\title{
MicroRNA-34a inhibits liver cancer cell growth by reprogramming glucose metabolism
}

\author{
HAI-FENG ZHANG ${ }^{*}$, YI-CHENG WANG ${ }^{*}$ and YI-DI HAN \\ Department of Hepatology, Qingdao No. 6 People's Hospital, Qingdao, Shandong 266033, P.R. China
}

Received August 6, 2017; Accepted December 5, 2017

DOI: $10.3892 / \mathrm{mmr} .2018 .8399$

\begin{abstract}
MicroRNAs (miRs) have been proposed as minimally invasive prognostic markers for various types of cancer, including liver cancer, which is one of the most common cancers worldwide. In the present study, the expression of miR-34a in human liver cancer tissues and cell lines was evaluated and the effects of miR-34a on cell proliferation, invasion and glycolysis in hepatocellular carcinoma (HCC) cells were determined. The results indicated that miR-34a was downregulated in human liver cancer tissues. Overexpression of miR-34a significantly inhibited liver cancer cell proliferation and clone formation. In terms of the underlying mechanism, miR-34a was indicated to negatively regulate the expression of lactate dehydrogenase A (LDHA), which consequently inhibited LDHA-dependent glucose uptake in the cancer cells, as well as cell proliferation and invasion. Collectively, these data suggest that miR-34a functions as a negative regulator of glucose metabolism and may serve as a novel marker for liver cancer prognosis.
\end{abstract}

\section{Introduction}

Liver cancer is one of the most common malignancies worldwide, with an increasing incidence, especially in China (1-3). Hepatocellular carcinoma (HCC) is the most common type of primary liver cancer; it is estimated to be the second leading cause of cancer-related death in developing countries and the sixth leading cause in developed countries (4-6). Despite recent advances in HCC management, including surgical resection, radiofrequency ablation, liver transplantation, and transcatheter arterial chemoembolization (TACE), the prognosis of most HCC patients remains poor. In addition, the molecular pathogenesis of HCC is not well understood.

Correspondence to: Dr Yi-Di Han, Department of Hepatology, Qingdao No. 6 People's Hospital, 9 Fushun Road, Qingdao, Shandong 266033, P.R. China

E-mail: yidihanmed@126.com

*Contributed equally

Key words: micrRNA-34a, liver cancer, glucose metabolism
Therefore, it is crucial to clarify the effector molecules and signaling pathways underlying HCC tumor progression and metastasis to develop novel therapeutic strategies and more effective treatments.

MicroRNAs (miRNAs/miRs) are endogenous small (19-25 nucleotide) non-coding RNAs. By binding to the 3'-untranslated regions (3'-UTRs) of targeted messenger RNAs (mRNAs) (7-9), miRNAs play important roles in post-transcriptional regulation and numerous biological processes such as proliferation, survival, and apoptosis $(10,11)$. Recently, several studies have found that miRNAs are also critical for cancer invasion and metastasis. Dysregulated expression of miRNAs is correlated with many human cancers, including HCC. In addition, miRNAs are emerging as promising diagnostic and prognostic markers for human cancers $(12,13)$.

miR-34a reportedly acts as a tumor suppressor in many cancers, including pancreatic cancer, prostate cancer, glioblastoma, colon cancer, and breast cancer (14-17). In pancreatic cancer, miR-34a inhibits stem cell self-renewal by downregulating Bcl-2 and Notch (18). In glioblastoma, miR-34a inhibits cell proliferation due to its regulation of the TGF- $\beta$ signaling network (19). Several recent studies have shown that the expression of miR-34a is dramatically decreased in clinical HCC specimens, suggesting that miR-34a represents a potential target for HCC treatment (20-22). However, the biological effect and underlying mechanism of miR-34a in HCC tumorigenesis and metastasis remains to be elucidated. Thus, further exploration of miR-34a is of utmost significance.

Lactate dehydrogenase A (LDHA) plays an important role in tumor cell metabolism (23-25). It has recently been reported that LDHA expression is correlated with progression and survival outcomes in multiple cancers, including renal cancer, gastric cancer, esophageal squamous cell carcinoma, and pancreatic cancer. Moreover, several oncogenes and deacetylases, including HIF-1 $\alpha$, SIRT2, and MYC, contribute to the regulation of LDHA expression and post-transcriptional modification $(26,27)$. Together, these studies indicate that LDHA could be a novel therapeutic target for multiple human cancers, including HCC.

In this study, we evaluated the expression of miR-34a in HCC tissues and cell lines. Furthermore, functional studies of the effects of miR-34a and LDHA on cell proliferation, invasion, and glycolysis in HCC cells were performed to explore the underlying connection between miR-34a and HCC. 


\section{Materials and methods}

Cell lines and culture. The HCC cell lines Huh7, HCCLM3, Hep3B, Mahlavu, SNU475, and human hepatocyte line L02 were obtained from the Cell Bank of the Chinese Academy of Sciences (Shanghai, China) and cultured in Dulbecco's modified Eagle's medium (DMEM) (Invitrogen; Thermo Fisher Scientific, Inc., Waltham, MA, USA) supplemented with $10 \%$ fetal bovine serum and $1 \%$ penicillin/streptomycin (Gibco; Thermo Fisher Scientific, Inc.) at $37^{\circ} \mathrm{C}$ in a humidified atmosphere with $5 \% \mathrm{CO}_{2}$.

Clinical samples. The study protocols were approved by the Ethics Committee of Qingdao No. 6 People's Hospital following the ethical standards outlined in the Declaration of Helsinki. Written informed consent was obtained from all patients. Tissue samples from 22 pairs of HCC tissues (HC) and their corresponding adjacent tissues (normal, located $\sim 2 \mathrm{~cm}$ apart) were collected in the Department of Hepatology, Qingdao No. 6 People's Hospital. The clinical profiles of the 22 participants in the study, such as gender, age, BMI, tumor size, and Child-Pugh class were described in Table I. Tissue samples were obtained and immediately stored in liquid nitrogen for further reverse transcription-quantitative polymerase chain reaction (RT-qPCR analysis.

RNA extraction and RT- $q P C R$. Total RNA was extracted from the tissues and cell lines using TRIzol reagent (Invitrogen; Thermo Fisher Scientific, Inc.). RT was performed using a Prime-Script RT Reagent kit (Takara Biotechnology Co., Ltd., Dalian, China) and RT-qPCR was performed using SYBR Premix Ex Taq (Takara Biotechnology Co., Ltd.). MicroRNA extraction was conducted using a MicroRNA Extraction kit (Tiangen Biotech Co., Ltd., Beijing, China) and RT-qPCR was performed with SYBR Premix Ex Taq (Takara Biotechnology Co., Ltd.) according to the manufacturer's protocol. GAPDH and RNU6B were used as normalizing controls for mRNA and miRNA quantification, respectively. The primers were as follows: miR-34a forward, 5'-UGGCAGUGUCUUAGC UGGUUGU-3' and reverse, 5'-AACCAGCUAAGACACUGC CAUU-3'; LDHA, forward, 5'-TTGGTCCAGCGTAACGTG AAC-3' and reverse, 5'-CCAGGATGTGTAGCCTTTGAG-3'. The $2^{-\Delta \Delta C q}$ method was used to determine relative expression levels.

Cell proliferation assay. HuH7 and HCCLM3 cells were seeded in 96-well plates and incubated for $24 \mathrm{~h}$ before being transfected with miR-34a or scrambled mimics. After the cells were incubated for another $48 \mathrm{~h}$, an MTT assay was performed according to the manufacturer's instructions (Molecular Probes; Thermo Fisher Scientific, Inc.). The absorbance at $570 \mathrm{~nm}$ was determined using a Spectra Max 250 spectrophotometer (Molecular Devices, Sunnyvale, CA, USA).

Foci formation assay. HuH7 and HCCLM3 cells were seeded in 6-well plates at a density of 2,000 cells per well and transfected with miR-34a or scrambled mimics. When the number of clones exceeded 50, the cells were stained with $0.06 \%$ crystal violet and the foci numbers were counted.
Cell invasion assay. A Transwell invasion assay was used to determine the invasion capacity of the tumor cells. Briefly, $\mathrm{HuH} 7$ and HCCLM3 cells were transfected with miR-34a or scrambled mimics, cultivated for $24 \mathrm{~h}$, and seeded onto the Matrigel-coated chambers (24-well; BD Biosciences, San Jose, CA, USA) in serum-free DMEM. DMEM containing 10\% FBS was added to the lower chamber. The matrix and non-invaded cells were removed after $48 \mathrm{~h}$ of incubation, while the invaded cells were fixed, stained, and counted.

Wound-healing assay. After transfection, $\mathrm{HuH} 7$ cells were seeded in 6-well plates and grown to $90 \%$ confluence. After $24 \mathrm{~h}$, linear scratch wounds were created using pipette tips and the cells were washed three times with PBS. The cells were then incubated in DMEM containing 5\% FBS. Cell movement at the wound site was monitored and photographed at 0 and $24 \mathrm{~h}$. The percentage of wound closure was analyzed and compared as described previously (24).

Measurement of glucose uptake and lactate production. To assess glucose uptake and lactate production, $\mathrm{HuH} 7$ and HCCLM3 cells were transfected with miR-34a or scrambled mimics, and the cell culture medium was collected $48 \mathrm{~h}$ after transfection. An Amplex ${ }^{\circledR}$ Red Glucose/Glucose Oxidase Assay kit (Invitrogen; Thermo Fisher Scientific, Inc.) was then used to measure glucose uptake, and a lactate assay kit (Sigma-Aldrich; Merck KGaA, Darmstadt, Germany) was used to determine lactate production.

Western blotting. HuH7 and HCCLM3 cells were transfected with either miR-34a or scrambled mimics. After $48 \mathrm{~h}$, protein was extracted using RIPA lysis buffer. Protein concentrations were quantified using a Protein BCA Assay kit (Pierce; Thermo Fisher Scientific, Inc.). The protein samples were separated by $10 \%$ SDS-PAGE then transferred to polyvinylidene difluoride membranes (EMD Millipore, Billerica, MA, USA). After blocking in 5\% skim milk for $1 \mathrm{~h}$ at room temperature, the membranes were incubated with antibodies against LDHA and $\beta$-actin (Affinity Biosciences, Columbus, $\mathrm{OH}$, USA) at $4^{\circ} \mathrm{C}$ overnight. A peroxidase-conjugated secondary antibody (dilution 1:1,500) was applied for $1 \mathrm{~h}$ at room temperature to visualize the target proteins. The target proteins were visualized using Western blotting detection reagents (Thermo Fisher Scientific, Inc.) and then exposed to X-ray film (Kodak, Inc., Rochester, NY, USA). The optical density (OD) (target proteins)/OD ( $\beta$-actin) was used to quantify protein expression.

LDHA-expressing vector. Full-length LDHA cDNA was purchased from GeneCopeia (Rockville, MD, USA) and sub-cloned into the expression vector pcDNA3.1(+) (GeneCopeia). The vector pcDNA3.1(+) was used as a negative control.

Statistical analysis. All in vitro experiments were performed in triplicate. The results are presented as means \pm standard deviation. Statistical comparisons between two groups were analyzed using t-tests and $\chi^{2}$ tests. Statistical comparisons between multiple groups were analyzed using one-way ANOVA followed by Newman-Keuls post-hoc comparison 
Table I. The clinical profiles of the 22 patients with hepatocellular carcinoma.

\begin{tabular}{lc}
\hline Clinical variable & No. of patients $(\mathrm{n}=22)$ \\
\hline Gender & 8 \\
Female & 14 \\
Male & \\
Age & 58.7 \\
Median & $40-79$ \\
Range & \\
HBV & 22 \\
Positive & 0 \\
Negative & \\
Tumor size & 13 \\
$\leq 5$ cm & 9 \\
$>5$ cm & \\
Child-Pugh class & 22 \\
A & 0 \\
B & \\
BMI & 21.2 \\
Median & $16-26.8$ \\
Range & \\
\hline
\end{tabular}

$\mathrm{HBV}$, hepatitis B virus; BMI, body mass index.

test. $\mathrm{P}<0.05$ was considered to indicate a statistically significant difference (SPSS 16.0; SPPS, Inc., Chicago, IL, USA).

\section{Results}

miR-34a was significantly downregulated in HCC cell lines and clinical specimens. A RT-qPCR analysis was employed to detect the expression of miR-34a. The results show that the expression of miR-34a was markedly downregulated in six different HCC cell lines (Huh7, HCCLM3, Hep3B, Mahlavu, and SNU475) compared to the human hepatocyte cell line L02 (Fig. 1A). To determine the expression of miR-34a in clinical specimens, HCC tissues (HC) and their matched adjacent normal tissues (Normal) were examined through RT-qPCR analysis. Compared with adjacent normal tissues, we found that $77.3 \%$ ( 17 of 22 patients, $\mathrm{P}<0.01$ ) of tumor tissues showed decreased miR-34a levels (Fig. 1B). Taken together, these results indicate that miR-34a is downregulated at a high frequency in HCC, and may be related to HCC carcinogenesis.

miR-34a inhibits cell proliferation and invasion. The expression of miR-34a was examined in HuH7 and HCCLM3 cells following transfection with miR-34a or scramble mimics. The RT-qPCR results show a significant increase in miR-34a $(\sim 94$ fold) in transfected cells compared to scramble or untreated cells $(\mathrm{P}<0.001)$ (Fig. 2A). To explore the biological effects of miR-34a in HCC, HuH7 and HCCLM3 cells were transfected with miR-34a or scramble mimics, and the number of cells was counted. The results show that ectopic expression of miR-34a significantly suppressed the proliferation of HuH7 and HCCLM3
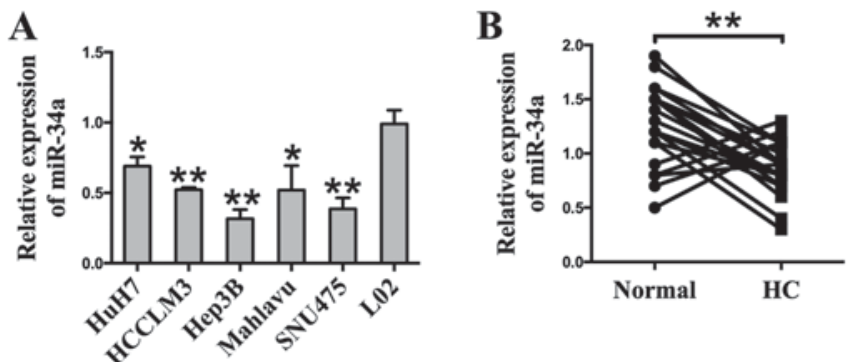

Figure 1. miR-34a is downregulated in liver cancer cell lines and clinical HCC specimens. (A) RT-qPCR analysis revealed the expression level of miR-34a in six HCC cell lines (Huh7, HCCLM3, HepG2, Hep3B, Mahlavu, and SNU475) and human hepatocyte line L02. (B) RT-qPCR was performed to determine the expression of miR-34a in 22 HCC tissues (HC) and their matched adjacent normal tissues (Normal). These results indicated that the expression of miR-34a was downregulated in HCC cell lines and clinical specimens. ${ }^{*} \mathrm{P}<0.05$ and ${ }^{* *} \mathrm{P}<0.01$ vs. L02.

cells in a time-dependent manner $(\mathrm{P}<0.05)$ (Fig. 2B); this was further confirmed by an MTT assay (Fig. 2C). In addition, the results of the foci formation assay show that the overexpression of miR-34a led to decreased foci formation of $\mathrm{HuH} 7$ and HCCLM3 cells $(\mathrm{P}<0.01)$ (Fig. 2D). To further explore the function of miR-34a in HCC, a Transwell invasion assay was performed. The results show that overexpression of miR-34a significantly inhibited invasion in HuH7 and HCCLM3 cells compared with the scramble group (Fig. 2E).

miR-34a inhibits glycolysis in HCC. To explore the role miR-34a in glycolysis in HCC, differences in metabolic parameters were detected after HuH7 and HCCLM3 cells were transfected with miR-34a or scramble mimics. The results show that overexpression of miR-34a significantly decreased glucose uptake $(\mathrm{P}<0.01)$ (Fig. 3A). In addition, the results indicate that miR-34a mimics induced a decrease in the production of extracellular lactate $(\mathrm{P}<0.05)$ (Fig. 3B). Taken together, these results suggest that the inhibition of glycolysis by miR-34a may be responsible for the suppression of migration and invasion in HCC cells.

miR-34a inhibits LDHA expression in HCC. LDHA is reportedly a direct target of miR-34a (28). To confirm this, RT-qPCR and Western blot analyses were performed. HuH7 and HCCLM3 cells were transfected with miR-34a or scramble mimics before examining LDHA expression. Our results show that cells transfected with miR-34a mimics showed a significant reduction in both the mRNA and protein levels of LDHA (Fig. 4A and B).

miR-34a inhibits LDHA-induced glycolysis, cell proliferation, and invasion. To confirm that miR-34a inhibited glycolysis, cell proliferation, and invasion of HCC cells by targeting LDHA, we transfected HuH7 and HCCLM3 cells with an LDHA-expressing vector, control vector, control vector + miR-34a mimics, control vector + a scrambled oligonucleotide, LDHA expressing vector + miR-34a mimics, or LDHA expressing vector + a scrambled oligonucleotide. The results show that overexpression of LDHA could increase HCC cell glucose uptake and lactate production, and that this effect was abolished by transfection with miR-34a mimics 


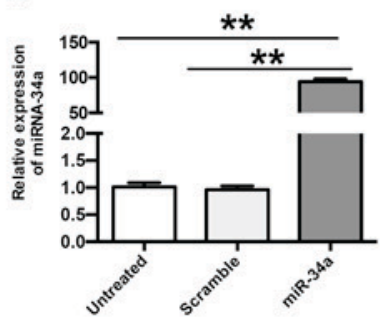

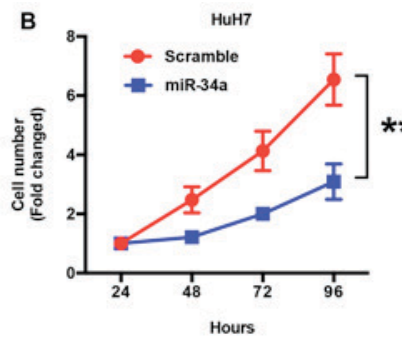

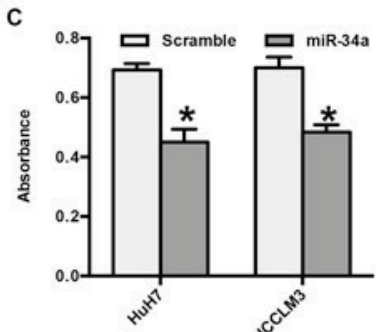

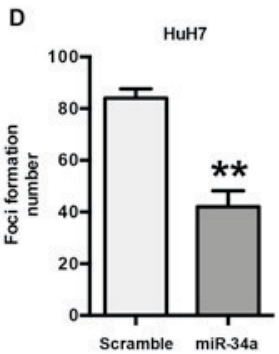
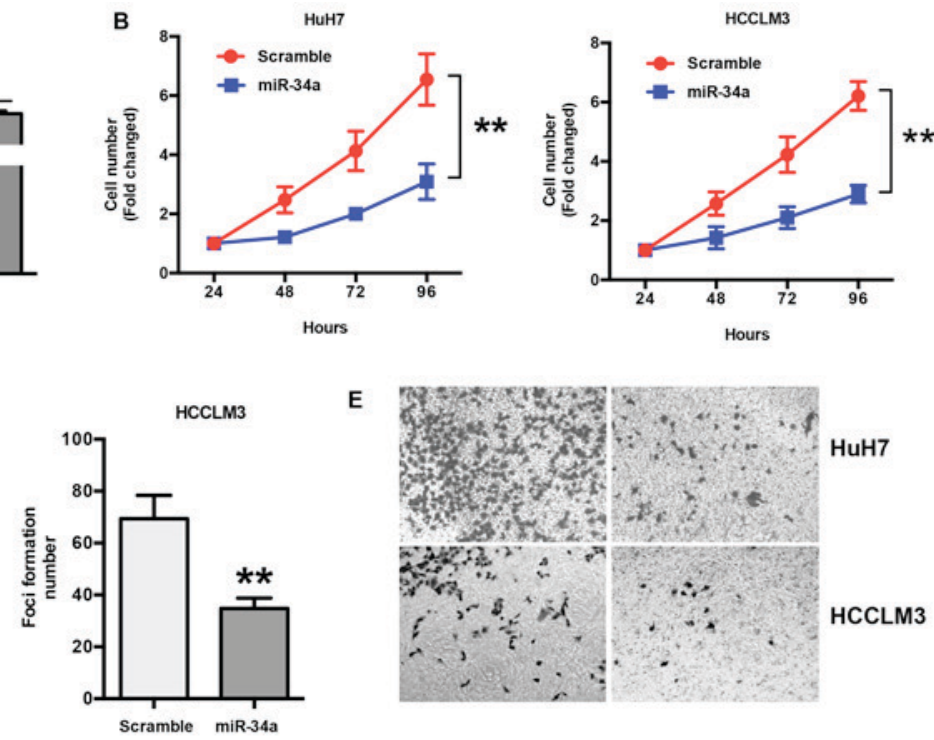

E

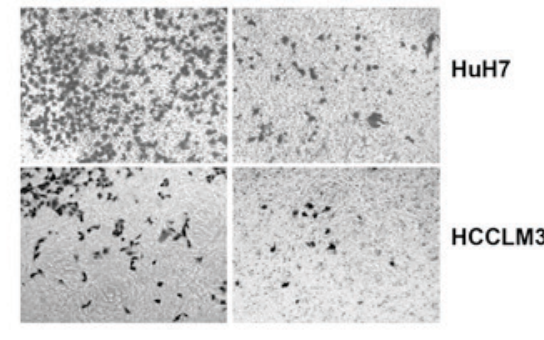

Figure 2. miR-34a inhibits cell proliferation and invasion. (A) The expression level of miR-34a was greatly increased by miR-34a mimics. ${ }^{* *} \mathrm{P}<0.01$. (B) The ectopic expression of miR-34a significantly suppressed the cell proliferation of HuH7 and HCCLM3 cells in a time dependent manner. ${ }^{* *}$ P $<0.01$ vs. Scramble. (C) The results of MTT assay showed miR-34a significantly suppressed cell proliferation in $48 \mathrm{~h}$ after transfection. * $\mathrm{P}<0.05$ vs. Scramble. (D) The results of foci formation assay showed that overexpression of miR-34a significantly decreased foci formation of HuH7 and HCCLM3 cells. ${ }^{*} \mathrm{P}<0.01$ vs. Scramble. (E) Representative images of three independent experiments are presented (magnification, x100). The results of transwell invasion assay showed that overexpression of miR-34a significantly inhibited cell invasion of HuH7 and HCCLM3 cells compared with the scramble group.
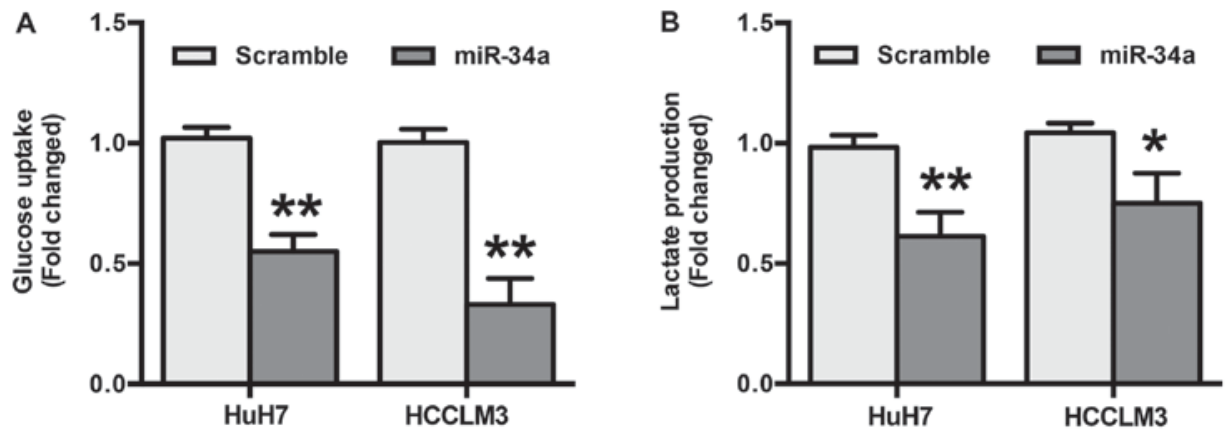

Figure 3. miR-34a inhibits glycolysis in hepatocellular carcinoma. (A) HuH7 and HCCLM3 cells were transfected with miR-34a or scramble mimics. The glucose uptake levels were measured after transfection. (B) HuH7 and HCCLM3 cells were transfected as described before and the lactate production levels were measured. The results showed that overexpression of miR-34a largely decreased glucose uptake and extracellular lactate production. ${ }^{*} \mathrm{P}<0.05$ and ${ }^{* * *} \mathrm{P}<0.01$ vs. Scramble.

(Fig. 5A and B). We also found that the overexpression of LDHA could promote cell proliferation, while miR-34a inhibited cell proliferation. The increased cell proliferation induced by LDHA was significantly repressed by miR-34a (Fig. 5C). Transwell invasion and wound-healing assays showed that miR-34a mimics decreased invasion capacity, which was increased by the overexpression of LDHA (Fig. 5D and E). Together, these findings demonstrate that miR-34a inhibits HCC glycolysis, and cell proliferation and invasion in vitro by targeting LDHA.

\section{Discussion}

Although outstanding advances in surgical techniques and radio-chemotherapy regimens have been achieved in recent decades, the prognosis for most patients with $\mathrm{HCC}$ remains poor. Therefore, novel therapeutic strategies are needed to treat HCC more effectively $(3,4,6)$.
Increasing evidence has demonstrated that miRNAs play a vital role in the pathogenesis, clinical metastasis, and progression of multiple cancers, including HCC (29-31). Fang et al (32) reported that miR-383 was downregulated in HCC tissues compared with their adjacent normal tissues. In addition, overexpression of miR-383 significantly suppressed cell proliferation and invasion, indicating that miR-383 may act as a tumor suppressor in HCC. Kota et al (33) demonstrated that systemic administration of miR-26a resulted in significant inhibition of HCC cell proliferation, and induced protection against disease progression without toxicity. Thus, understanding the crucial role of miRNAs in HCC may provide novel diagnostic, prognostic, and therapeutic potential.

miR-34a is a member of the highly conserved miR-34 family. It is located at the chromosome lp36 locus, which is particularly susceptible to molecular events that may disturb the balance of proliferation and apoptosis $(34,35)$. miR-34a is also one of multiple recently discovered miRNAs that are 

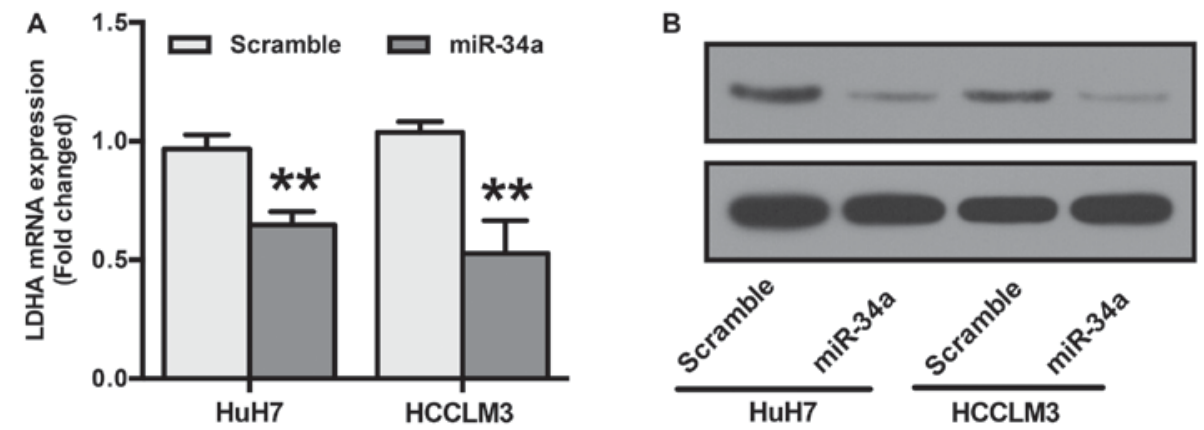

Figure 4. LDHA is a target of miR-34a. (A) HuH7 and HCCLM3 cells were transfected with miR-34a or scramble mimics. Overexpression of miR-34a significantly downregulated the levels of LDHA mRNA. (B) Overexpression of miR-34a significantly inhibited the LDHA protein expression. ${ }^{* *} \mathrm{P}<0.01$ vs. Scramble.

A

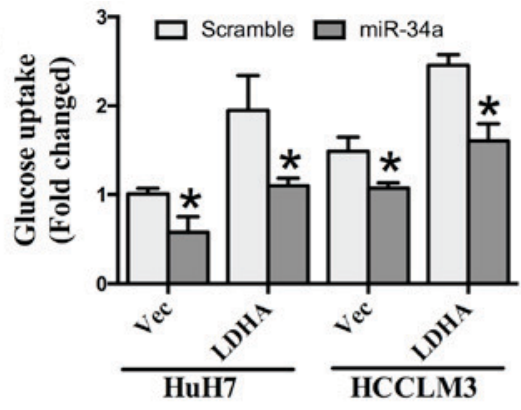

C

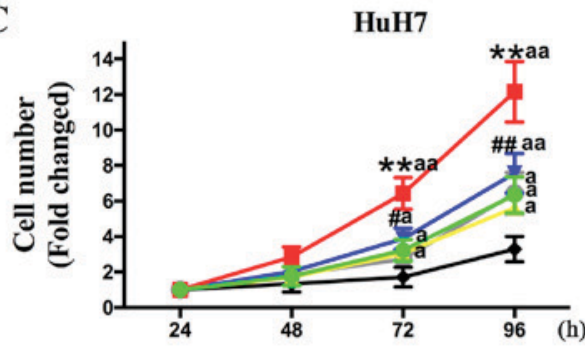

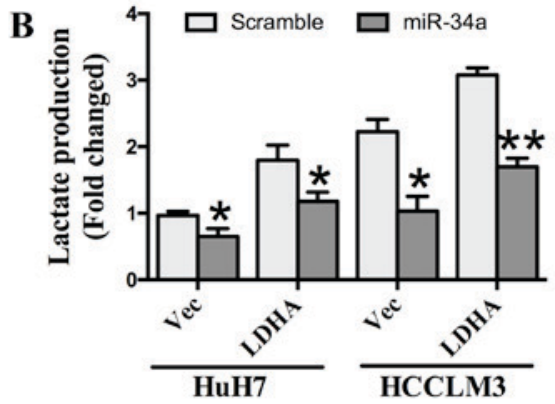

HCCLM3

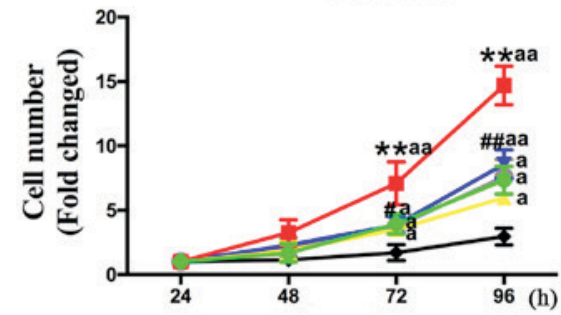

miR-34a

miR-34a+LDHA

D
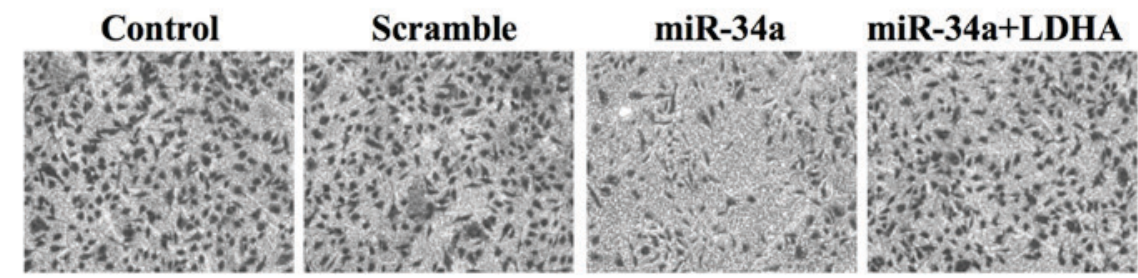

$\mathbf{E}$

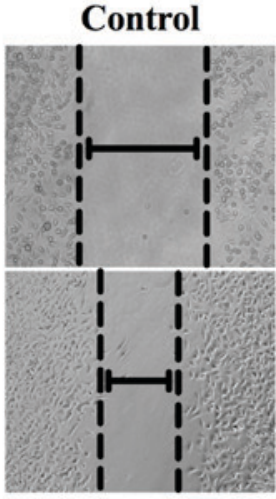

Scramble

miR-34a

miR-34a+LDHA
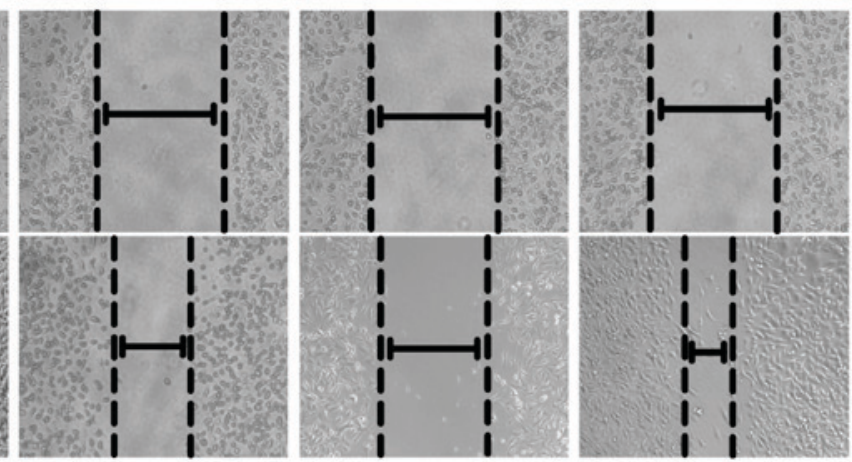

Figure 5. LDHA-induced glycolysis and cell proliferation can be inhibited by miR-34a. (A) Overexpression of LDHA could increase HCC cell glucose uptake, which was abrogated by miR-34a after cotransfection with miR-34a mimics. ${ }^{*} \mathrm{P}<0.05$ vs. Scramble. (B) Increased lactate production by LDHA was abrogated by miR-34a. ${ }^{*} \mathrm{P}<0.05$ and ${ }^{* *} \mathrm{P}<0.01$ vs. Scramble. (C) HuH7 and HCCLM3 cells were transfected as described before. After transfection, the increased cell proliferation of HuH7 and HCCLM3 cells by LDHA was suppressed by miR-34a in a time-dependent manner. ${ }^{* *} \mathrm{P}<0.01$ vs. Vec; ${ }^{~} \mathrm{P}<0.05$ and ${ }^{\# \#} \mathrm{P}<0.01$ vs. LDHA; ${ }^{2} \mathrm{P}<0.05$ and aa $\mathrm{P}<0.01$ vs. miR-34a. (D) miR-34a mimics reversed the invasion capacity which was increased by overexpressed LDHA (magnification, $\mathrm{x} 200$ ). (E) miR-34a mimics reversed the migration capacity which was increased by overexpressed LDHA (magnification, x100). 
regulated by p53 (17,36). Studies have shown that miR-34a is an important molecule that inhibits the growth of many cancers. Decreased expression of miR-34a has been found in pancreatic cancer, cervical cancer, prostate cancer, glioblastoma, colon cancer, esophageal squamous cell carcinoma, breast cancer, and lung cancer (37-39). In cancers like pancreatic cancer and glioblastoma, miR-34a was reported to inhibit cell proliferation and induce apoptosis by downregulating Bcl-2 and Notch or the TGF- $\beta$ signaling network $(18,19,40)$. However, the role of miR-34a in the pathogenesis of HCC is unclear.

Several studies have shown that miR-34a may act as an oncogene (41). Pineau et al (42) found that miR-34a was highly expressed in liver cancer, and was positively related to HCC progression. In a tamoxifen-induced mouse liver cancer model, Pogribny et al (43) found that miR-34 expression was increased. However, more and more studies have recently shown that the expression of miR-34a is dramatically decreased in clinical HCC specimens, suggesting that miR-34a is a potential target for HCC treatment (20-22). Dang et al (44) detected miR-34a expression in 60 HCC tissues and adjacent normal tissues and found that miRNA-34a expression in HCC tissues was significantly lower than in normal tissues. Moreover, a miR-34a mimic was reported to inhibit HCC cell growth and induce apoptosis. However, the mechanism remains unknown. In this study, we found that miR-34a was downregulated in human liver cancer tissues, and that the upregulation of miR-34a inhibits liver cancer cell proliferation, migration, and invasion, consistent with previous studies $(20-22,44)$. Moreover, we found that miR-34a negatively regulates the expression of LDHA in HCC cell lines, which consequently inhibits LDHA-dependent glucose uptake in cancer cells, leading to reduced cell proliferation and invasion.

Aberrant metabolism has been shown to play an important role in the progression and metastasis of multiple cancers (45-47). Among a series of enzymes involved in cancer metabolism, LDHA is reported to be of vital importance and is involved in proliferation and glycolysis in gastric cancer, breast cancer, and pancreatic cancer $(23,25,27,48)$. Indeed, some references have already proved that miR-34a negatively regulates the expression level of LDHA in various cancers $(23,25,28,49)$. By performing luciferase reporter assays, or cloning of 3'-UTRs, miR-34a was reported to have direct role on LDHA mRNA stability in various cell lines $(23,28)$, including HCC cells (50). In a previous study based on HCC cells, Wang et al reported that miR-34a specifically bind to the 3'-UTR region of LDHA, acting as a negative regulator (50). In this study, we confirmed that LDHA is also involved in HCC metabolism, and an LDHA-expressing vector significantly improved $\mathrm{HCC}$ cell glycolysis, proliferation, migration, and invasion. Furthermore, we also confirmed that LDHA may be a target gene for miR-34a. The function of miR-34a in HCC may be partly due to its regulation of LDHA and the subsequent reprogramming of glucose metabolism. The increased cell glycolysis, proliferation, and invasion triggered by LDHA was effectively inhibited by miR-34a in HCC cell lines, which indicates that the miR-34a-LDHA axis could be a promising therapeutic target for more effective HCC treatment.
To the best of our knowledge, this is the first study of the effect of miR-34a on HCC glucose metabolism. However, some limitations of our study should be noted. First, this was an in vitro study that used clinical samples, but it lacked an in vivo evaluation of the potential impact of miR-34a. Second, the carcinogenesis of HCC is complicated, which means miR-34a may also target other genes. In addition, although the results of this study showed that LDHA was downregulated in miR-34a transfected $\mathrm{HuH} 7$ and HCCLM3 cells, further analysis about the direct role of miR-34a on LDHA mRNA stability was not conducted, which may decrease the robustness of the conclusion of our study. Nevertheless, our study provides useful insight into the effect of miR-34a on cell proliferation, invasion, and glycolysis in HCC cell lines. Future studies are needed.

In summary, the results of this study suggest that miR-34a inhibits HCC glycolysis, cell proliferation, and invasion in vitro by targeting LDHA. miR-34a functions as a negative regulator of glucose metabolism, which may serve as a novel marker for liver cancer prognosis.

The results of this study suggest that miR-34a inhibits HCC glycolysis, cell proliferation, and invasion in vitro by targeting LDHA.

\section{References}

1. Zhong GC, Liu Y, Chen N, Hao FB, Wang K, Cheng JH, Gong JP and Ding $X$ : Reproductive factors, menopausal hormone therapies and primary liver cancer risk: A systematic review and dose-response meta-analysis of observational studies. Hum Reprod Update 23: 126-138, 2016.

2. Nio K, Yamashita T and Kaneko S: The evolving concept of liver cancer stem cells. Mol Cancer 16: 4, 2017.

3. Rao CV, Asch AS and Yamada HY: Frequently mutated genes/pathways and genomic instability as prevention targets in liver cancer. Carcinogenesis 38: 2-11, 2017.

4. Rabinel P, Dousse D, Muscari F and Suc B: Management of liver cancer. The Surgeon's point of view. Rep Pract Oncol Radiother 22: 176-180, 2017.

5. Sia D, Villanueva A, Friedman SL and Llovet JM: Liver cancer cell of origin, molecular class, and effects on patient prognosis. Gastroenterology 152: 745-761, 2017.

6. Stroehl YW, Letzen BS, van Breugel JM, Geschwind JF and Chapiro J: Intra-arterial therapies for liver cancer: Assessing tumor response. Expert Rev Anticancer Ther 17: 119-127, 2017.

7. Ma J, Lin J, Qian J, Qian W, Yin J, Yang B, Tang Q, Chen X, Wen X, Guo H and Deng Z: miR-378 promotes the migration of liver cancer cells by down-regulating Fus expression. Cell Physiol Biochem 34: 2266-2274, 2014.

8. Pang F, Zha R, Zhao Y, Wang Q, Chen D, Zhang Z, Chen T, Yao M, Gu J and He X: miR-525-3p enhances the migration and invasion of liver cancer cells by downregulating ZNF395. PLoS One 9: e90867, 2014.

9. Yin J, Bai Z, Song J, Yang Y, Wang J, Han W, Zhang J, Meng H, Ma X, Yang Y, et al: Differential expression of serum miR-126, miR-141 and miR-21 as novel biomarkers for early detection of liver metastasis in colorectal cancer. Chin J Cancer Res 26: 95-103, 2014.

10. Guo W, Qiu Z, Wang Z, Wang Q, Tan N, Chen T, Chen Z, Huang S, Gu J, Li J, et al: miR-199a-5p is negatively associated with malignancies and regulates glycolysis and lactate production by targeting hexokinase 2 in liver cancer. Hepatology 62: 1132-1144, 2015.

11. Liu HB, Hua Y and Jin ZX: Effects of MicroRNA-132 transfection on the proliferation and apoptosis of human liver cancer cells in vitro and in vivo. Zhongguo Yi Xue Ke Xue Yuan Xue Bao 37: 30-36, 2015.

12. Lu Z, Zhang W, Gao S, Jiang Q, Xiao Z, Ye L and Zhang X: miR-506 suppresses liver cancer angiogenesis through targeting sphingosine kinase 1 (SPHK1) mRNA. Biochem Biophys Res Commun 468: 8-13, 2015. 
13. Sun B, Li J, Shao D, Pan Y, Chen Y, Li S, Yao X, Li H, Liu W, Zhang M, et al: Adipose tissue-secreted miR-27a promotes liver cancer by targeting FOXO1 in obese individuals. Onco Targets Ther 8: 735-744, 2015.

14. Dong P, Xiong Y, Watari H, Hanley SJ, Konno Y, Ihira K, Yamada T, Kudo M, Yue J and Sakuragi N: miR-137 and miR-34a directly target Snail and inhibit EMT, invasion and sphere-forming ability of ovarian cancer cells. J Exp Clin Cancer Res 35: 132, 2016.

15. Chen W, Liu Y, Liang X, Huang Y and Li Q: Chondroitin sulfate-functionalized polyamidoamine as a tumor-targeted carrier for miR-34a delivery. Acta Biomater 57: 238-250, 2017.

16. Chen Y, Sun P, Guo X and Gao A: miR-34a, a promising novel biomarker for benzene toxicity, is involved in cell apoptosis triggered by 1,4-benzoquinone through targeting Bcl-2. Environ Pollut 221: 256-265, 2017.

17. Lin Y, Shen J, Li D, Ming J, Liu X, Zhang N, Lai J, Shi M, Ji Q and Xing Y: miR-34a contributes to diabetes-related cochlear hair cell apoptosis via SIRT1/HIF-1 $\alpha$ signaling. Gen Comp Endocrinol 246: 63-70, 2017

18. Ji Q, Hao X, Zhang M, Tang W, Yang M, Li L, Xiang D, Desano JT, Bommer GT, Fan D, et al: MicroRNA miR-34 inhibits human pancreatic cancer tumor-initiating cells. PLoS One 4: e6816, 2009.

19. Genovese G, Ergun A, Shukla SA, Campos B, Hanna J, Ghosh P, Quayle SN, Rai K, Colla S, Ying $\mathrm{H}$, et al: microRNA regulatory network inference identifies miR-34a as a novel regulator of TGF- $\beta$ signaling in glioblastoma. Cancer Discov 2: 736-749, 2012.

20. Tryndyak VP, Ross SA, Beland FA and Pogribny IP: Down-regulation of the microRNAs miR-34a, miR-127, and miR-200b in rat liver during hepatocarcinogenesis induced by a methyl-deficient diet. Mol Carcinog 48: 479-487, 2009.

21. Chen Q, Li L, Tu Y, Zheng LL, Liu W, Zuo XY, He YM, Zhang SY, Zhu W, Cao JP, et al: miR-34a regulates apoptosis in liver cells by targeting the KLF4 gene. Cell Mol Biol Lett 19: 52-64, 2014.

22. Wang XP, Zhou J, Han M, Chen CB, Zheng YT, He XS and Yuan XP: MicroRNA-34a regulates liver regeneration and the development of liver cancer in rats by targeting Notch signaling pathway. Oncotarget 8: 13264-13276, 2017.

23. Kaller M, Liffers ST, Oeljeklaus S, Kuhlmann K, Röh S, Hoffmann R, Warscheid B and Hermeking H: Genome-wide characterization of miR-34a induced changes in protein and mRNA expression by a combined pulsed SILAC and microarray analysis. Mol Cell Proteomics 10: M111.010462, 2011.

24. DuJY,WangLF,WangQand YuLD:miR-26binhibitsproliferation, migration, invasion and apoptosis induction via the downregulation of 6-phosphofructo-2-kinase/fructose-2,6-bisphosphatase-3 driven glycolysis in osteosarcoma cells. Oncol Rep 33: 1890-1898, 2015.

25. Wang J, Wang H, Liu A, Fang C, Hao J and Wang Z: Lactate dehydrogenase A negatively regulated by miRNAs promotes aerobic glycolysis and is increased in colorectal cancer. Oncotarget 6 : 19456-19468, 2015.

26. Song K, Kwon H, Han C, Zhang J, Dash S, Lim K and Wu T: Active glycolytic metabolism in CD133(+) hepatocellular cancer stem cells: Regulation by MIR-122. Oncotarget 6: 40822-40835, 2015.

27. Ullmann P, Qureshi-Baig K, Rodriguez F, Ginolhac A, Nonnenmacher Y, Ternes D, Weiler J, Gabler K, Bahlawane C, Hiller K, et al: Hypoxia-responsive miR-210 promotes self-renewal capacity of colon tumor-initiating cells by repressing ISCU and by inducing lactate production. Oncotarget 7: 65454-65470, 2016

28. Xiao X, Huang X, Ye F, Chen B, Song C, Wen J, Zhang Z, Zheng G, Tang H and Xie X: The miR-34a-LDHA axis regulates glucose metabolism and tumor growth in breast cancer. Sci Rep 6: 21735, 2016.

29. Yang Q, Jiang W, Zhuang C, Geng Z, Hou C, Huang D, Hu L and Wang X: microRNA-22 downregulation of galectin-9 influences lymphocyte apoptosis and tumor cell proliferation in liver cancer. Oncol Rep 34: 1771-1778, 2015.

30. Retraction notice to microarray analysis of microRNA expression in liver cancer tissues and normal control [GENE 523/2 (2014) 158-60]. Gene 578: 137, 2016.

31. Liu C, Wang C, Wang J and Huang H: miR-1297 promotes cell proliferation by inhibiting RB1 in liver cancer. Oncol Lett 12: 5177-5182, 2016
32. Fang Z, He L, Jia H, Huang Q, Chen D and Zhang Z: The miR-383-LDHA axis regulates cell proliferation, invasion and glycolysis in hepatocellular cancer. Iran J Basic Med Sci 20: 187-192, 2017.

33. Kota J, Chivukula RR, O'Donnell KA, Wentzel EA, Montgomery CL, Hwang HW, Chang TC, Vivekanandan P, Torbenson M, Clark KR, et al: Therapeutic microRNA delivery suppresses tumorigenesis in a murine liver cancer model. Cell 137: 1005-1017, 2009.

34. Cui H, Ge J, Xie N, Banerjee S, Zhou Y, Antony VB, Thannickal VJ and Liu G: miR-34a inhibits lung fibrosis by inducing lung fibroblast senescence. Am J Respir Cell Mol Biol 56: 168-178, 2017.

35. Fu BC, Lang JL, Zhang DY, Sun L, Chen W, Liu W, Liu KY, Ma CY, Jiang SL, Li RK and Tian H: Suppression of miR-34a expression in the myocardium protects against ischemia-reperfusion injury Through SIRT1 protective pathway. Stem Cells Dev 26: 1270-1282, 2017

36. Jiang L and Hermeking H: miR-34a and miR-34b/c suppress intestinal tumorigenesis. Cancer Res 77: 2746-2758, 2017

37. Liu YP, Hu H, Xu F and Wen JJ: Relation of miR-34a expression in diffuse large B cell lymphoma with clinical prognosis. Zhongguo Shi Yan Xue Ye Xue Za Zhi 25: 455-459, 2017 (In Chinese).

38. Maroni P, Puglisi R, Mattia G, Care A, Matteucci E, Bendinelli P and Desiderio MA: In bone metastasis miR-34a-5p absence inversely correlates with Met expression, while Met oncogene is unaffected by miR-34a-5p in non-metastatic and metastatic breast carcinomas. Carcinogenesis 38: 492-503, 2017.

39. Song C, Lu P, Sun G, Yang L, Wang Z and Wang Z: miR-34a sensitizes lung cancer cells to cisplatin via p53/miR-34a/MYCN axis. Biochem Biophys Res Commun 482: 22-27, 2017.

40. Chen AH, Qin YE, Tang WF, Tao J, Song HM and Zuo M: miR-34a and miR-206 act as novel prognostic and therapy biomarkers in cervical cancer. Cancer Cell Int 17: 63, 2017.

41. Sukata T, Sumida K, Kushida M, Ogata K, Miyata K, Yabushita S and Uwagawa S: Circulating microRNAs, possible indicators of progress of rat hepatocarcinogenesis from early stages. Toxicol Lett 200: 46-52, 2011

42. Pineau P, Volinia S, McJunkin K, Marchio A, Battiston C, Terris B, Mazzaferro V, Lowe SW, Croce CM and Dejean A: miR-221 overexpression contributes to liver tumorigenesis. Proc Natl Acad Sci USA 107: 264-269, 2010.

43. Pogribny IP, Tryndyak VP, Boyko A, Rodriguez-Juarez R, Beland FA and Kovalchuk O: Induction of microRNAome deregulation in rat liver by long-term tamoxifen exposure. Mutat Res 619: 30-37, 2007.

44. Dang Y, Luo D, Rong M and Chen G: Underexpression of miR-34a in hepatocellular carcinoma and its contribution towards enhancement of proliferating inhibitory effects of agents targeting c-MET. PLoS One 8: e61054, 2013.

45. Chen B, Li H, Zeng X, Yang P, Liu X, Zhao X and Liang S: Roles of microRNA on cancer cell metabolism. J Transl Med 10: 228 , 2012.

46. Zhao E, Maj T, Kryczek I, Li W, Wu K, Zhao L, Wei S, Crespo J, Wan S, Vatan L, et al: Cancer mediates effector T cell dysfunction by targeting microRNAs and EZH2 via glycolysis restriction. Nat Immunol 17: 95-103, 2016.

47. Xu W, Zhang Z, Zou K, Cheng Y, Yang M, Chen H, Wang H, Zhao J, Chen P, He L, et al: miR-1 suppresses tumor cell proliferation in colorectal cancer by inhibition of Smad3-mediated tumor glycolysis. Cell Death Dis 8: e2761, 2017.

48. Han RL, Wang FP, Zhang PA, Zhou XY and Li Y: miR-383 inhibits ovarian cancer cell proliferation, invasion and aerobic glycolysis by targeting LDHA. Neoplasma 64: 244-252, 2017.

49. Zhang R, Su J, Xue SL, Yang H, Ju LL, Ji Y, Wu KH, Zhang YW, Zhang YX, Hu JF and Yu MM: HPV E6/p53 mediated down-regulation of miR-34a inhibits Warburg effect through targeting LDHA in cervical cancer. Am J Cancer Res 6: 312-320, 2016.

50. Wang J, Yan S, Zhang W, Zhang H and Dai J: Integrated proteomic and miRNA transcriptional analysis reveals the hepatotoxicity mechanism of PFNA exposure in mice. J Proteome Res 14: 330-341, 2015.

This work is licensed under a Creative Commons Attribution-NonCommercial-NoDerivatives 4.0 International (CC BY-NC-ND 4.0) License. 